\title{
Poster Presentation
}

\section{MS13.P07}

RNA Biogenesis: Mechanism and Evolution of RNase III

\author{
$\underline{\mathrm{X} . \mathrm{Ji}^{1}}$ \\ ${ }^{1}$ National Cancer Institute, Macromolecular Crystallography Laboratory, Frederick, USA
}

RNase III represents a family of dsRNA-specific endoribonucleases required for RNA maturation and gene regulation. Bacterial RNase III and eukaryotic Rnt1p, Drosha, and Dicer are representative members of the family. The bacterial enzyme possesses a single RNase III domain (RIIID) followed by a dsRNA-binding domain (dsRBD); Rnt1p is defined by the presence of an N-terminal domain (NTD), a RIIID, and a dsRBD; Drosha contains N-terminal P-rich and RS-rich domains followed by two RIIIDs and a dsRBD; and Dicer possesses $\mathrm{N}$-terminal helicase, DUF283, and PAZ domains followed by two RIIIDs and a dsRBD. It is the N-terminal extension beyond the RIIID that distinguishes eukaryotic RNase IIIs from the bacterial enzyme. My lab has been studying the structure and mechanism of RNase III enzymes since 1996. We have reported a total of eleven crystal structures of bacterial RNase III in complex with dsRNA at various catalytic stages of the enzyme, including the first structure of a catalytically meaningful RNase III-RNA complex (Gan et al., Cell, 124:355-366, 2006), and thereby well characterized the mechanism of action for the bacterial enzyme (Court et al., Annu Rev Genet, 47:405-431, 2013). We have also determined the crystal structure of yeast Rnt1p post-cleavage complex, the first structure of a eukaryotic RNase III complexed with RNA in a catalytically meaningful manner (Liang et al., Molecular Cell, 54:431-444, 2014, featured article on the issue cover). Strikingly, the NTD and dsRBD of Rnt1p function as two rulers for substrate selection. This unusual mechanism represents an example of the evolution of substrate selectivity and provides a framework for understanding the catalytic mechanism of eukaryotic RNase IIIs, including Drosha and Dicer.

[1] Gan J, Tropea JE, Austin BP, Court DL, Waugh DS, Ji X. Structural insight into the mechanism of double-stranded RNA processing by ribonuclease III. Cell. 124: 355-66, 2006., [2] Court DL, Gan J, Liang Y, Shaw GX, Tropea JE, Costantino N, Waugh DS, Ji X. RNase III: Genetics and Function; Structure and Mechanism. Annu Rev Genet. 47: 405-431, 2013., [3] Liang Y, Lavoie M, Comeau M, Abou Elela S, Ji X. Structure of a Eukaryotic RNase III Postcleavage Complex Reveals a Double-Ruler Mechanism for Substrate Selection. Mol. Cell. 54: 431-444, 2014.

Keywords: RNase III, dsRNA processing, RNA biogenesis 